\title{
Enhanced Thermal Stability of Gold and Silver Nanorods by Thin Surface Layers
}

\author{
Yuriy Khalavka, Christian Ohm, Litao Sun, Florian Banhart,* and Carsten Sönnichsen* \\ Institute for Physical Chemistry, University of Mainz, Jakob-Welderweg 11, 55128 Mainz, Germany
}

Received: July 5, 2007; In Final Form: August 1, 2007

\begin{abstract}
Using in situ transmission electron microscopy, we find that a carbon shell governs the morphological transitions of gold and silver nanorods upon heating. Encapsulated Ag nanorods show a surprising nonuniform sublimation behavior starting from one side and leaving behind the shell. Uncovered gold nanorods transform their shape to spheres well below the bulk melting temperature through surface diffusion, which is prevented by a thin carbon shell.
\end{abstract}

The thermodynamic properties of materials are known to differ from their bulk values when particles have nanometer dimensions. ${ }^{1,2}$ Responsible for this size effect is the large surface-to-volume ratio of nanoparticles, the sometimes unique crystal structure of nanoparticles, and the chemical nature of their surface. Gold nanoparticles were among the first artificially created inorganic nanostructures, ${ }^{3,4}$ and their response to heating has been extensively studied. ${ }^{5,6}$ The thermal stability of noble metal particles is of interest both for fundamental reasons and because gold and silver nanoparticles show a unique optical response (plasmons) making them useful for a range of optical applications. Rod-shaped gold and silver particles show much stronger plasmon resonances compared to spherical particles ${ }^{7}$ and have been the subject of intense research after the discovery of a wet-chemical route for their production. ${ }^{8,9}$ The temperature stability of gold nanorods has been experimentally studied using various structural analysis techniques before and after heat treatment, for example, induced by ultrafast lasers, ${ }^{10-13}$ and has been subject to extensive theoretical modeling. ${ }^{14-17}$ Spherical silver nanoparticles have received considerably less attention, ${ }^{18}$ and silver rod melting has not been studied so far, despite the fact that silver nanoparticles are in many aspects superior to gold particles. Silver shows about 30\% higher thermal and electrical conductivity compared to gold, and the plasmon resonance lifetime is about $20 \%$ longer. ${ }^{19,20}$ Because of the large fraction of surface atoms, the behavior of small particles upon heating (in particular morphological changes toward the equilibrium shape) should be strongly influenced by the presence of a surface-covering shell. Here, we study the thermally induced transformation of rod-shaped silver and gold particles when the particles are covered by a thin carbon shell.

In situ experiments are carried out in a transmission electron microscope (TEM) (FEI Tecnai F-30) equipped with a field emission gun and spectrometers for energy dispersive X-ray spectroscopy and electron energy loss spectroscopy (EELS). A high-temperature specimen stage (Gatan) allows heating of the samples up to $1570 \mathrm{~K}$ during inspection in the microscope. The temperature of the samples is measured with a thermocouple

* Corresponding authors. E-mail: (C.S.) soennichsen@uni-mainz.de; (F.B.) banhart@uni-mainz.de. welded to the specimen support plate. The silver nanorods are produced by our version ${ }^{21}$ of the seeded growth method in concentrated surfactant solution of Jana et al. ${ }^{22}$ and the gold nanorods by the method of Nikoobakht and El-Sayed. ${ }^{8}$ Both methods are very similar and the particles are produced in a concentrated aqueous solution of a surfactant (cetyl-trimetylammonium bromide, CTAB). The silver nanorods appear only in a small yield among spherical and other irregularly shaped nanocrystals. For a typical experiment, we dilute $100 \mu \mathrm{L}$ of the as-prepared nanoparticle suspension in $1 \mathrm{~mL}$ of deionized water, centrifuge for $7 \mathrm{~min}$ at $7000 \mathrm{rpm}(5500 \mathrm{~g})$, and remove the supernatant. This is repeated with fresh water twice. At the end, the particles are resuspended in $50 \mu \mathrm{L}$ of water and a drop placed on a piece of Parafilm. A carbon-coated TEM grid, pretreated for $30 \mathrm{~s}$ in oxygen plasma to make it hydrophilic, is briefly placed on top of the drop and then dried for $2 \mathrm{~h}$. The samples are then either directly used for an in situ heating experiment or stored under vacuum for later use.

The samples of gold nanorods are sometimes placed again in oxygen plasma to remove any remaining organic layers on the particle surface. Unfortunately, the silver nanorods do not sustain a treatment with oxygen or argon plasma and show a clearly visible surface reaction with air after 1 day of exposure (Supporting Information, Figure S1). It is, therefore, likely that despite the extensive washing with water, a layer of surfactants from the synthesis procedure is still surrounding the silver nanorods. Uncovered silver particles could not be produced by our techniques.

Performing the in situ heating experiment with silver nanorods leads to a surprising observation: the silver nanorods are stable against heating to temperatures close to the bulk melting point, show no or little shape transformation, but eventually a strongly anisotropic sublimation (Figure 1 and Supporting Information, Figure S2). Upon heating, the particles first show a weakening of the internal symmetric defect and twinning plane structures (Figure 1, first 3 images), a slightly rounded outer shape, but no observable mass loss. At some point (here after $30 \mathrm{~min}$ at $885 \mathrm{~K}$ ), a shell around the particles (which is hardly visible before sublimation of the Ag particle) seems to break at one of the particle tips and the particles rapidly lose material at this 

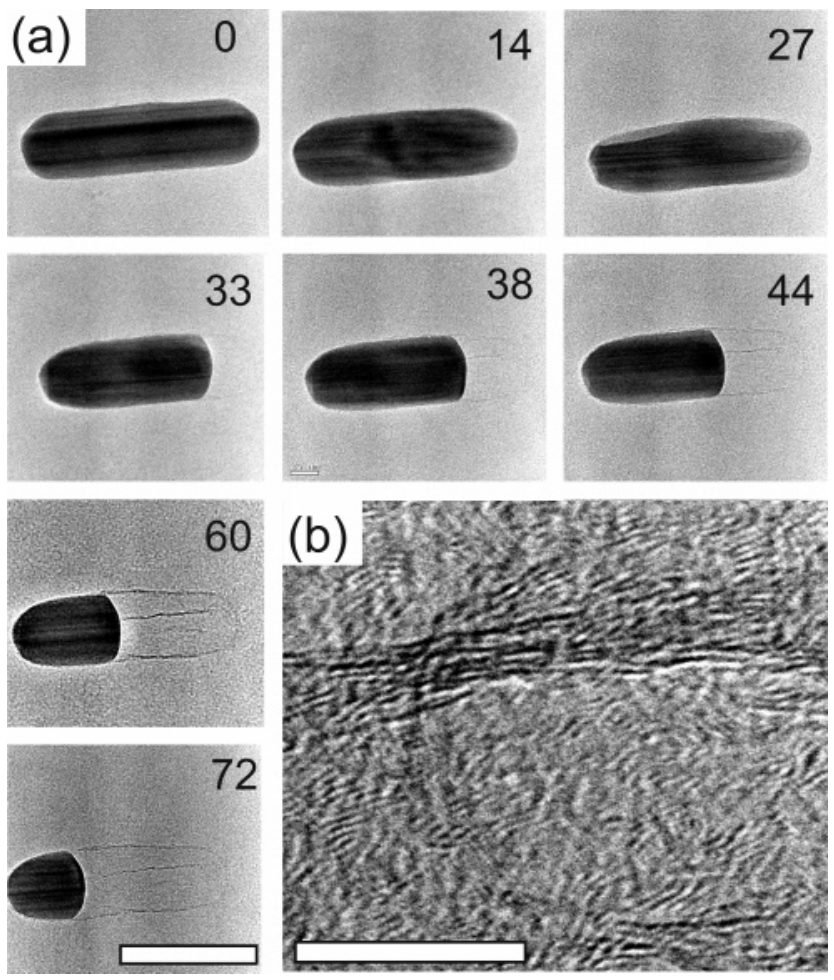

Figure 1. (a) Sublimation of a silver nanorod at $885 \mathrm{~K}$. Time as indicated (in minutes). The rod shrinks starting from the right leaving behind a shell structure. Solid part shows fringes indicating crystalline structure scalebars $100 \mathrm{~nm}$. (b) High-resolution image of the shell structure showing the typical appearance of graphitic filaments. Scalebar $10 \mathrm{~nm}$.

end (Figure 1, image 4). A thin cagelike structure with the original particle dimension is left behind (Figure 1, last 6 images).

The thin shell around the silver particles is presumably of graphitic nature (Figure 1b). The spacing between the fringes is approximately $0.34 \mathrm{~nm}$, and the lattice image is typical for graphite. An elemental analysis by EELS is difficult due to the signal from the carbon grid. The graphite shells could originate from organic residues on the surface that graphitized at high temperatures. It was shown by Chou and $\mathrm{McAtee}^{23}$ that a carbon layer produced by decomposition of amino-organic compounds on porous materials is stable in oxygen-free atmosphere up to $1073 \mathrm{~K}$. The melting or decomposing temperature of possible silver compounds is too low to give the observed high-thermal stability $\left(\mathrm{Ag}_{2} \mathrm{O}, 373 \mathrm{~K} ; \mathrm{AgBr}, 705 \mathrm{~K} ; \mathrm{AgBrO}_{3}, 633 \mathrm{~K} ; \mathrm{AgCl}\right.$, $728 \mathrm{~K} ; \mathrm{AgClO}_{3}, 503 \mathrm{~K}$ ). ${ }^{24}$ Formation of $\mathrm{Ag}_{2} \mathrm{~S}$ (melting point $1098 \mathrm{~K})^{24}$ is unlikely in our sample preparation procedure.

The mechanism of the anisotropic sublimation process of silver nanorods becomes more explicit by observing a larger sample area and measuring particle sizes at different points in time while keeping the sample at $990 \mathrm{~K}$ (Figure 2 and Supporting Information, Figure S3). From these measurements, we calculate the particle volume assuming hemispherically capped cylinders. Figure $2 \mathrm{c}$ shows the time development of these calculated volumes for some representative particles in the sample area. Most particles, regardless whether they are rodshaped or not, show a more or less constant volume loss rate after a small delay at the start of the experiment. At the end, this volume loss somewhat slows down. The linear volume loss observed on rod-shaped particles supports the view that the particles sublimate through an opening of a shell surrounding them (Figure 2b). Otherwise, one would expect the rate to be proportional to the surface area (in the case of sublimation into

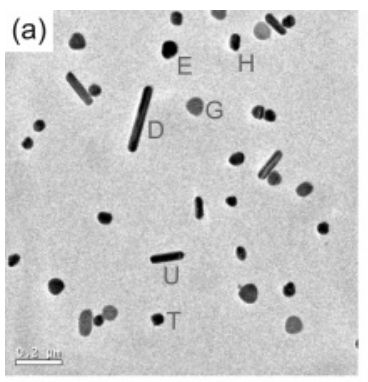

(b)

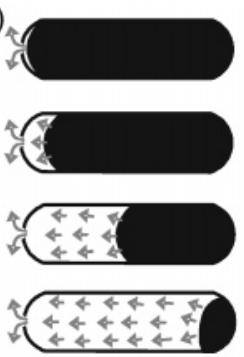

(c)

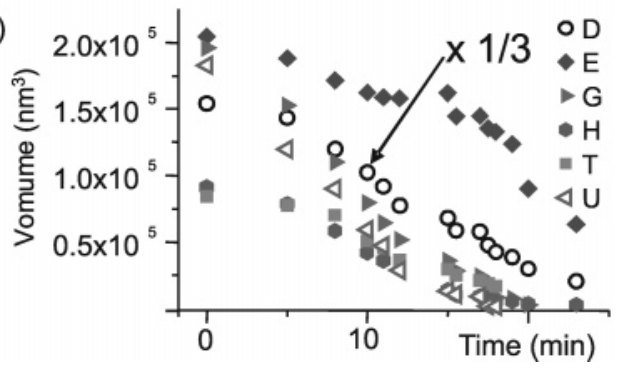

Figure 2. Silver nanorods on carbon film at $990 \mathrm{~K}$ continuously observed over $23 \mathrm{~min}$. The particles are identified (a) and their small and long axis length determined for each point in time. The mass or volume loss of the particles should be linear in time assuming an open shell around the particles (b). We calculate particle volumes assuming hemispherical-capped cylinders and show some representative values in (c). The volume decreases monotonously for rods (open symbols) and spherical particles (closed symbols).

the vacuum) or the circumference (in the case of surface diffusion of metal atoms on the substrate). The breaking of the shell at the tips of the rods may be due to the higher vapor pressure of strongly curved surfaces. ${ }^{25}$ Using values of 1.13 to $6.5 \mathrm{~J} / \mathrm{m}^{2}$ for the surface energy reported in ref 26 , we estimate a vapor pressure increase by a factor of 2 to 25 .

To study the role of the material, we investigated samples of gold nanorods. The pristine gold nanorods were free from any surface shells, which were removed by extensive washing and plasma cleaning. Figure 3a shows a sequence of TEM images of a gold nanorod gradually changing its shape upon heating. The morphological transformation starts at temperatures well below the bulk-melting point of gold $(1337 \mathrm{~K})$ with conformation changes on time scales of hours starting at about $650 \mathrm{~K}$. Shape transformations of gold rods have been reported for temperatures as low as $523 \mathrm{~K}^{10}$. Similar but slightly slower behavior was observed on rods that were not plasma cleaned (Supporting Information, Figure S4). We have also coated gold nanorods artificially by a thin amorphous carbon shell in an evaporation apparatus (resistive heating). Figure $3 \mathrm{~b}$ shows that carbon-covered gold nanorods are stable above $940 \mathrm{~K}$. They slowly change their shape only close to the bulk-melting temperature, that is, at considerably higher temperatures than pristine gold nanorods. It is apparent that a carbon shell effectively prevents shape transformations. However, gold nanorods never show the strongly anisotropic sublimation behavior as observed with silver nanorods, regardless of the way the gold rods are deposited: "as prepared", plasma cleaned, or even coated with an extra carbon shell.

To confirm the different behaviors of silver and gold particles, we have prepared a mixture of gold nanorods and silver particles containing a few silver nanorods. Both kinds of nanoparticles are prepared using similar chemicals and, in particular, the same surfactant molecule (CTAB). The different behavior is clearly observable in the response to heating: shape transformation toward round particles and slow sublimation of gold nanorods, no shape transformation, and rapid anisotropic sublimation of 

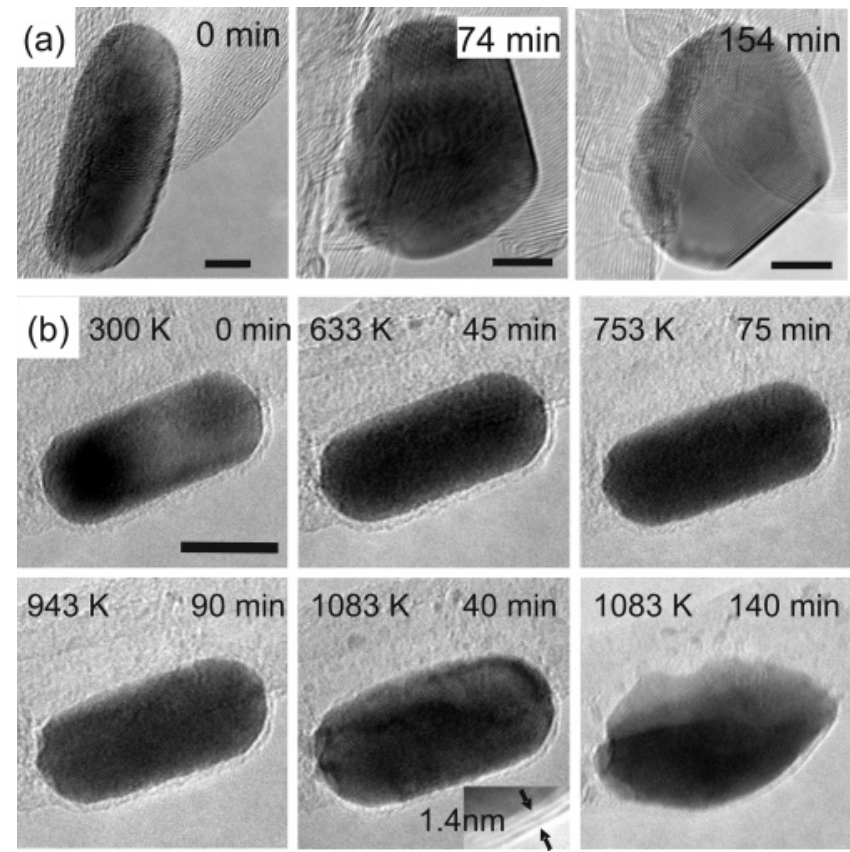

Figure 3. (a) Bare gold nanorods at $773 \mathrm{~K}$. Time as indicated, scalebar $50 \mathrm{~nm}$. The crystal lattice is visible throughout. (b) Gold nanorods coated by an amorphous carbon shell, which slowly graphitizes. The inset shows 3-4 basal layers of graphite on the particle surface. Temperature and time as indicated. Scalebar is $20 \mathrm{~nm}$.
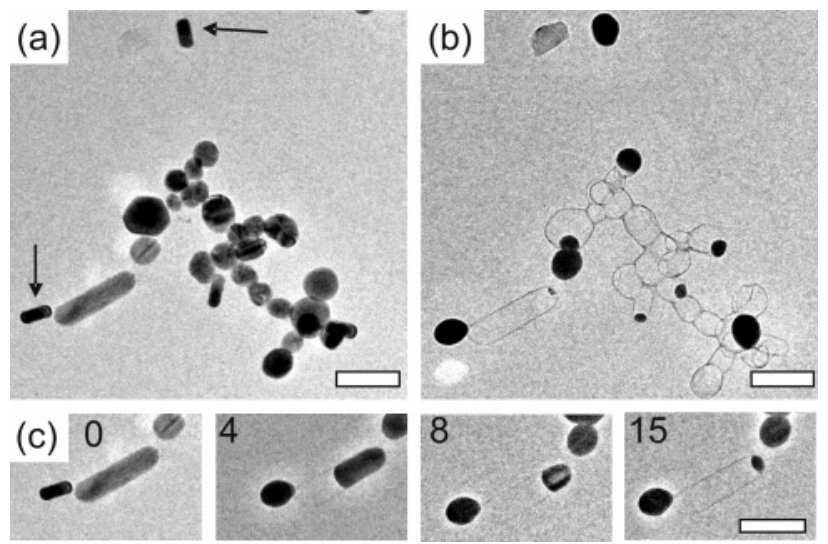

Figure 4. Thermal transformation of gold and silver nanoparticles observed simultaneously on the same substrate (carbon film). (a,b) Show overviews at room-temperature and after $30 \mathrm{~min}$ of heating at $960 \mathrm{~K}$, respectively. Particles identified as gold nanorods by contrast and size are marked by arrows. (c) Details for a gold and a silver nanorod at the times indicated in the images (in min). All scalebars are $100 \mathrm{~nm}$.

silver nanorods from one side leaving behind a thin shell (Figure $4 \mathrm{a}-\mathrm{c})$.

It has already been demonstrated that coherent and rigid graphitic shells around nanometer-sized metal crystals are able to stabilize the crystals considerably. ${ }^{27-29}$ In this study, however, the shells are graphitic but very thin. We have to assume that the graphitic layers here are not rigid because of the presence of defects (noncoherent layers, Figure 1b). Nevertheless, we observe that the shells have a strong influence on shape transformations, and it has to be explained how transformations of the particles toward the equilibrium shape can be influenced by the presence of such a surface layer. Whereas bulk diffusion of metal atoms should lead to shape changes irrespective of the presence of a nonrigid surface layer, surface diffusion of atoms could be suppressed by a shell. Thus, the mechanism of shape transformations of uncovered metal rods has to be due
TABLE 1: Material Properties of Gold and Silver and the Estimation of Vapor Pressure and Evaporation Rate for Solid Materials

\begin{tabular}{lrcc}
\hline & $\mathrm{Ag}$ & $\mathrm{Au}$ & ref \\
\hline bulk melting temperature $(\mathrm{K})$ & 1234 & 1337 & 24 \\
enthalpy of fusion $\Delta_{\text {fus }} H(\mathrm{~kJ} / \mathrm{mol})$ & 11 & 13 & 24 \\
enthalpy of evap $\Delta_{\text {vap }} H(\mathrm{~kJ} / \mathrm{mol})$ & 258 & 324 & 30 \\
temp with vapor press $1 \mathrm{~Pa}(\mathrm{~K})$ & 1283 & 1646 & 24 \\
vapor pressure at $990 \mathrm{~K}(\mu \mathrm{Pa})$ & 566 & 0.0820 & \\
evap rate at $990 \mathrm{~K}\left(\right.$ atoms $\left./ \mathrm{nm}^{2} / \mathrm{s}\right)$ & 5 & 0.0005 &
\end{tabular}

to diffusion of surface atoms from the regions of high curvature at the ends of the rods toward the straight cylindrical faces. The process will continue until a spherical shape of the particle is attained, and random surface diffusion no longer leads to shape changes. Covering nonspherical nanoparticles by a surface layer could hence be an efficient way of preventing undesired thermal transformations of the particles toward the equilibrium shape.

The fast and anisotropic sublimation behavior of silver particles, which was not observed for gold particles, may be explained by the different vapor pressures of the two materials. Other relevant material properties of gold and silver (like the melting point) are very similar (Table 1). The rate of evaporation $R$ of a flat surface may be estimated by $R=1 / 4 \mathrm{p} / \mathrm{k} T v_{\mathrm{th}}$, where the thermal velocity $v_{\text {th }}=(8 \mathrm{kT} / \pi \mathrm{m})^{1 / 2}$, the vapor pressure is $p$, the temperature is $T$, and the atomic mass is $m .{ }^{31}$ The vapor pressure $p$ at temperature $T=960 \mathrm{~K}$, which is the temperature of the experiment with both materials on the same substrate (Figure 4), can be calculated using the Clausius-Clapeyron equation: $R \ln \left(p / p_{0}\right)=\Delta_{\text {sub }} H\left(1 / T-1 / T_{0}\right)$, where the gas constant is $R$, the enthalpy of sublimation $\Delta_{\text {sub }} H=\Delta_{\text {fus }} H+$ $\Delta_{\text {vap }} H$, and tabulated values are for the pressure $p_{0}$ at temperature $T_{0}$. The resulting estimated values for the vapor pressure and the rate of evaporation shown in Table 1 clearly show a huge difference in evaporation rate, which explains the observed difference in sublimation behavior. Partial surface melting, for which we have found no evidence here, may further enhance the mass loss. Because the vapor pressure of $\mathrm{Ag}$ is only $566 \mu \mathrm{Pa}$ at the temperature of this experiment $(960 \mathrm{~K})$, but nevertheless the graphitic shells open to let off the pressure, we can conclude that the graphitic shells do not act as rigid high-pressure cells such as in other experiments on carbonencapsulated metals. ${ }^{28}$ Therefore, the sublimation of silver must be suppressed by the graphitic shell that covers the metal surface.

The observed difference between gold and silver nanorods shows how a slightly different surface stability and surface chemistry affects the nanocrystal stability of otherwise similar materials to an unexpected degree. The thermal stabilization of gold rods by a thin surface coating may help in high-power applications (e.g., enhancement of nonlinear optical effects) by pushing the thermal destruction threshold considerably.

Acknowledgment. The authors wish to thank R. Würfel for technical assistance and A. Krasheninnikov for helpful discussions. Y.K., C.O., and L.S. contributed equally and are in alphabetical order.

Supporting Information Available: Additional figures as mentioned in the text and a discussion of the role of the substrate material. This material is available free of charge via the Internet at http://pubs.acs.org.

\section{References and Notes}

(1) Couchman, P. R.; Jesser, W. A. Nature 1977, 269, 481. 
(2) Yeshchenko, O. A.; Dmitruk, I. M.; Alexeenko, A. A.; Dmytruk, A. M. Phys. Rev. B. 2007, 75, 085434.

(3) Faraday, M. Phil. Trans. Royal Soc. London 1857, 147, 145.

(4) Zsigmondy, R.; Thiessen, P. A. Das Kolloide Gold; Akademische Verlagsgesellschaft M.B.H.: Göttingen, Germany, 1925; Vol. 1.

(5) Cleveland, C. L.; Luedtke, W. D.; Landman, U. Phys. Rev. B 1999, 60,5065 .

(6) Koga, K.; Ikeshoji, T.; Sugawara, K. Phys. Rev. Lett. 2004, 92, 115507.

(7) Sönnichsen, C.; Franzl, T.; Wilk, T.; von Plessen, G.; Feldmann, J.; Wilson, O.; Mulvaney, P. Phys. Rev. Lett. 2002, 88, 077402.

(8) Nikoobakht, B.; El-Sayed, M. A. Chem. Mater. 2003, 15, 1957. (9) Jana, N. R.; Gearheart, L.; Murphy, C. J. Adv. Mater. 2001, 13, 1389.

(10) Petrova, H.; Juste, J. P.; Pastoriza-Santos, I.; Hartland, G. V.; LizMarzan, L. M.; Mulvaney, P. Phys. Chem. Chem. Phys. 2006, 8, 814

(11) Plech, A.; Kotaidis, V.; Gresillon, S.; Dahmen, C.; von Plessen, G. Phys. Rev. B. 2004, 70, 195423.

(12) Link, S.; El-Sayed, M. A. J. Chem. Phys. 2001, 114, 2362.

(13) Link, S.; Burcla, C.; Mohamed, M. B.; Nikoobakht, B.; El-Sayed, M. A. J. Phys. Chem. A 1999, 103, 1165.

(14) de Bas, B. S.; Ford, M. J.; Cortie, M. B. J. Phys.-Condes. Matter 2005, 18, 55 .

(15) Wautelet, M.; Dauchot, J. P.; Hecq, M. J. Phys.: Condens. Matter 2003, 15, 3651 .

(16) Bai, X. M.; Li, M. Nano Lett. 2006, 6, 2284.

(17) Wang, Y. T.; Teitel, S.; Dellago, C. Nano Lett. 2005, 5, 2174.
(18) Lee, J. G.; Lee, J.; Tanaka, T.; Mori, H. Phys. Rev. Lett. 2006, 96 075504

(19) Sönnichsen, C.; Franzl, T.; Wilk, T.; von Plessen, G.; Feldmann, J. New J. Phys. 2002, 4, 93.

(20) Sönnichsen, C. Plasmons in metal nanostructures; Cuvillier Verlag: Göttingen, Germany, 2001.

(21) Boleininger, J.; Kurz, A.; Reuss, V.; Sönnichsen, C. Phys. Chem. Chem. Phys. 2006, 8, 3824 .

(22) Jana, N. R.; Gearheart, L.; Murphy, C. J. Chem. Commun. 2001, 617.

(23) Chou, Ch.Ch.; McAttee, J. L., Jr. Clays Clay Miner. 1969, 17, 339.

(24) CRC Handbook of Chemistry and Physics; 85th ed.; CRC Press: Boca Raton, FL, 2004.

(25) Nanda, K. K.; Kruis, F. E.; Fissan, H. Phys. Rev. Lett. 2002, 89 , 256103.

(26) Nanda, K. K.; Maisels, A.; Kruis, F. E.; Fissan, H.; Stappert, S. Phys. Rev. Lett. 2003, 91, 106102.

(27) Banhart, F.; Grobert, N.; Terrones, M.; Charlier, J. C.; Ajayan, P. M. Int. J. Mod. Phys. B 2001, 15, 4037.

(28) Banhart, F.; Hernandez, E.; Terrones, M. Phys. Rev. Lett. 2003, 90,185502 .

(29) Li, J. X.; Banhart, F. Adv. Mater. 2005, 17, 1539.

(30) Speight, J. G. Lange's Handbook of Chemistry; 16th ed.; McGrawHill: New York, 2005.

(31) Hagelaar, G. J. M.; Pitchford, L. C. J. Anal. At. Spectrom. 2002 , 17,1408 . 